\title{
Bayesian Estimation Based on Progressively Type-II Censored Samples from Compound Rayleigh Distribution
}

\author{
Rashad Mohamed EL-Sagheer \\ Department of Mathematics, Faculty of Science, Al-Azhar University, Nasr City 11884, Cairo, Egypt \\ Rashadmath@yahoo.com \\ Mohamed Ahsanullah \\ Department of Management Sciences, Rider Univesity, New Jersey, USA \\ ahsan@rider.edu \\ Received 19 June 2014 \\ Accepted 7 April 2015
}

\begin{abstract}
This paper considers inference under progressive type-II censoring scheme with a compound Rayleigh failure time distribution. The maximum likelihood (ML) and the Bayes estimators for the two unknown parameters of the compound Rayleigh distribution (CRD) distribution are derived. A Bayesian approach using Markov chain Monte Carlo (MCMC) method to generate from the posterior distributions and in turn computing the Bayes estimators are developed. Point estimation and confidence intervals based on maximum likelihood and bootstrap methods are also proposed. The approximate Bayes estimators have been obtained under the assumptions of informative and non-informative priors. An example with the real data is discussed to illustrate the proposed methods. Finally, we made comparisons between the maximum likelihood and different Bayes estimators using a Monte Carlo simulation study.

Keywords: Compound Rayleigh distribution (CRD); Progressive type-II censoring; Bootstrap methods; Bayesian and non-Bayesian approach; Markov chain Monte Carlo (MCMC); Gibbs and Metropolis sampler.
\end{abstract}

2000 Mathematics Subject Classification: 62N05, 62F10

\section{Introduction}

In the past several decades, censoring is very common in life tests. There are many situations in life-testing and reliability studies in which the experimenter may be unable to obtain complete information on failure times of all experimental items. There are also situations wherein the removal of items prior to failure is pre-planned in order to reduce the cost and time associated with testing. The most common censoring schemes are type-I and type-II censoring, but the conventional type-I and type-II censoring schemes do not have the flexibility of allowing removal of items at points other than the terminal point of the experiment. For this reason, we consider a more general censoring scheme called progressive type-II right censoring. It can be described as follows. Suppose 
that $n$ independent items are put on a life test with continuous identically distributed failure times $X_{1}, X_{2}, \ldots, X_{n}$. Suppose further that a censoring scheme $\left(R_{1}, R_{2}, \ldots, R_{m}\right)$ is previously fixed such that immediately following the first failure $X_{1}, R_{1}$ surviving items are removed from the experiment at random, and immediately following the second failure $X_{2}, R_{2}$ surviving items are removed from the experiment at random. This process continues until, at the time of the $m$ th observed failure $X_{m}$, the remaining $R_{m}$ surviving items are removed from the test. The $m$ ordered observed failure times denoted by $X_{1: m: n}^{\left(R_{1}, \ldots, R_{m}\right)}, X_{2: m: n}^{\left(R_{1}, \ldots, R_{m}\right)}, \ldots, X_{m: m: n}^{\left(R_{1}, \ldots, R_{m}\right)}$ are called progressively type-II right censored order statistics of size $m$ from a sample of size $n$ with progressive censoring scheme $\left(R_{1}, R_{2}, \ldots, R_{m}\right)$. It is clear that $n=m+\sum_{i=1}^{m} R_{i}$. The special case when $R_{1}=R_{2}=\cdots=R_{m-1}=0$ so that $R_{m}=n-m$ is the case of conventional type-II right censored sampling. Also when $R_{1}=R_{2}=\cdots=R_{m}=0$, so that $m=n$, the progressively type-II right censoring scheme reduces to the case of no censoring (ordinary order statistics). Many authors have discussed inference under progressive type-II censored using different lifetime distributions, see for example, [7,5,20,22,17,8] and [16]. A thorough overview of the subject of progressive censoring is given in [4], and in the excellent review article by [3].

In Bayesian approach, we need to integrate over the posterior distribution and the problem is that the integrals are usually impossible to evaluate analytically. Markov chain Monte Carlo (MCMC) technique is a Monte Carlo integration method which draws samples from the target posterior distribution. MCMC methodology provided a convenient and efficient way to sample from complex, high-dimensional statistical distributions. Recently, application of the MCMC method to the estimation of parameters or some other vital properties about statistical models is very common. Green et al. [12] using the MCMC method for estimating the three parameters Weibull distribution, and they showed that the MCMC method is better than the ML method, when given a proper prior distribution of the parameters. As a generalization of the two parameter Weibull model, Gupta et al. [13] gave a complete Bayesian analysis of the Weibull extension model using MCMC simulation and complete sample. Recently, Mahmoud et al.[18] using Markov chain Monte Carlo to Study the estimation of the coefficient of variation.

The compound Rayleigh distribution (CRD) is a special case of the three-parameter Burr typeXII distribution. The two-parameter version of this distribution was studied by several authors, such as [1], [2] among others. The two parameter compound Rayleigh distribution $\operatorname{CRD}(\alpha, \beta)$ provides a population model which is useful in several areas of statistics, including life testing and reliability. The probability density function (pdf), and the cumulative distribution function (cdf) of the $\operatorname{CRD}(\alpha, \beta)$ are given, respectively, by

$$
f(x ; \alpha, \beta)=2 \alpha \beta^{\alpha} x\left(\beta+x^{2}\right)^{-(\alpha+1)}, x>0, \alpha, \beta>0,
$$

and

$$
F(x ; \alpha, \beta)=1-\left(1+\frac{x^{2}}{\beta}\right)^{-\alpha}, x>0, \alpha, \beta>0,
$$

where $\alpha$ and $\beta$ are the shape and the scale parameters respectively.

In this paper we consider the Bayesian inference of the shape and scale parameters for progressive type-II censored data when both parameters are unknown. We assumed that the shape parameter $\alpha$ and the scale parameter $\beta$ have the gamma prior and they are independently distributed. As expected in this case also, the Bayes estimates can not be obtained in closed form. We propose 
to use the Gibbs sampling procedure to generate MCMC samples, and then using the MetropolisHastings (MH) algorithms, we obtain the Bayes estimates of the unknown parameters. We perform some simulation experiments to see the behavior of the proposed Bayes estimators and compare their performances with the maximum likelihood estimators (MLEs).

The rest of the paper is organized as follows. In the next section, the ML estimators of the unknown parameters and approximate confidence intervals are presented. The corresponding parametric bootstrap confidence intervals for the parameters are given in Section 3. In Section 4, we cover Bayes estimates and construction of credible intervals using the MCMC techniques. In Section 5, for illustrative purposes, we performed a real data analysis. Comparisons among estimators are investigated through Monte Carlo simulations in Section 6. Finally, conclusions appear in Section 7.

\section{Maximum likelihood estimation}

If the failure times of the items originally on test with progressive censoring scheme $\left(R_{1}, R_{2}, \ldots, R_{m}\right)$ are from a continuous population with probability density function $f(x)$ and cumulative distribution function $F(x)$, then the joint probability density function of a progressively type-II censored sample $\underline{x}=X_{1: m: n}^{\left(R_{1}, \ldots, R_{m}\right)}, X_{2: m: n}^{\left(R_{1}, \ldots, R_{m}\right)}, \ldots, X_{m: m: n}^{\left(R_{1}, \ldots, R_{m}\right)}$ of size $m$ from a sample of size $n$ is given by

$$
f_{x_{1}, x_{2}, \ldots, x_{m}}\left(x_{1}, x_{2}, \ldots, x_{m}\right)=A \prod_{i=1}^{m} f\left(x_{i} ; \alpha, \beta\right)\left[1-F\left(x_{i} ; \alpha, \beta\right)\right]^{R_{i}}
$$

where $x_{i}$ is used instead of $X_{i: m: n}^{\left(R_{1}, \ldots, R_{m}\right)}, R \geq 0, i=1,2, \ldots, m$ and

$$
A=n\left(n-1-R_{1}\right)\left(n-2-R 1-R_{2}\right) \ldots\left(n-\sum_{i=1}^{m-1}\left(R_{i}+1\right)\right),
$$

and $f(),. F($.$) are the same as defined before in (1.1) and (1.2) respectively. The likelihood function$ can be written as

$$
L(\underline{x} ; \alpha, \beta)=A(2 \alpha)^{m}\left(\prod_{i=1}^{m} \frac{x_{i}}{\left(\beta+x_{i}^{2}\right)}\right) \exp \left[-\alpha \sum_{i=1}^{m}\left(R_{i}+1\right) \log \left(1+\frac{x_{i}^{2}}{\beta}\right)\right],
$$

where $A$ is defined in (2.2), Therefore ignoring the additive constant the log-likelihood function may be written as

$$
\ell(\underline{x} ; \alpha, \beta)=m \log \alpha+\sum_{i=1}^{m} \log x_{i}-\sum_{i=1}^{m} \log \left(\beta+x_{i}^{2}\right)-\alpha \sum_{i=1}^{m}\left(R_{i}+1\right) \log \left(1+\frac{x_{i}^{2}}{\beta}\right) .
$$

Assuming that the parameters $\alpha$ and $\beta$ are unknown, the MLEs of the parameters $\alpha$, and $\beta$ are obtained by solving the following likelihood equations simultaneously:

$$
\widehat{\alpha}=m\left[\sum_{i=1}^{m}\left(R_{i}+1\right) \log \left(1+\frac{x_{i}^{2}}{\hat{\beta}}\right)\right]^{-1},
$$

and

$$
\widehat{\alpha} \sum_{i=1}^{m}\left(R_{i}+1\right) \frac{x_{i}^{2} / \hat{\beta}^{2}}{1+\left(x_{i}^{2} / \hat{\beta}\right)}-\sum_{i=1}^{m} \frac{1}{\left(\hat{\beta}+x_{i}^{2}\right)}=0
$$


Since Eq. (2.6) cannot be solved analytically some numerical methods such as Newton-Raphson method must be employed.to solve (2.6) and get the MLE, $\widehat{\beta}$, and hence $\widehat{\alpha}$, by using (2.5).

The asymptotic variance-covariance matrix of the MLE for parameters $\alpha$, and $\beta$ is given by elements of the inverse of the Fisher information matrix

$$
\mathbf{I}_{i j}=E\left[-\frac{\partial^{2} L}{\partial \alpha \partial \beta}\right], i, j=1,2 .
$$

Unfortunately, the exact mathematical expressions for the above expectations are very difficult to obtain. Therefore, we give the approximate (observed) asymptotic varaince-covariance matrix for the MLE, which is obtained by dropping the expectation operator $E$

$$
\left[\begin{array}{cc}
-\frac{\partial^{2} \ell(\underline{x} ; \alpha, \beta)}{\partial \alpha^{2}} & -\frac{\partial^{2} \ell(\underline{x} ; \alpha, \beta)}{\partial \alpha \partial \beta} \\
-\frac{\partial^{2} \ell(\underline{x} ; \alpha, \beta)}{\partial \beta \partial \alpha} & -\frac{\partial^{2} \ell(\underline{x} ; \alpha, \beta)}{\partial \beta^{2}}
\end{array}\right]_{(\alpha=\widehat{\alpha}, \beta=\widehat{\beta})}^{-1}=\left[\begin{array}{cc}
\operatorname{var}(\widehat{\alpha}) & \operatorname{cov}(\widehat{\alpha}, \widehat{\beta}) \\
\operatorname{cov}(\widehat{\beta}, \widehat{\alpha}) & \operatorname{var}(\widehat{\beta})
\end{array}\right]
$$

with

$$
\begin{gathered}
\frac{\partial^{2} \ell(\underline{x} ; \alpha, \beta)}{\partial \alpha^{2}}=-\frac{m}{\alpha^{2}} \\
\frac{\partial^{2} \ell(\underline{x} ; \alpha, \beta)}{\partial \alpha \partial \beta}=\frac{\partial^{2} \ell(\underline{x} ; \alpha, \beta)}{\partial \beta \partial \alpha}=\sum_{i=1}^{m}\left(R_{i}+1\right) \frac{x_{i}^{2} / \beta^{2}}{1+\left(x_{i}^{2} / \beta\right)} \\
\frac{\partial^{2} \ell(\underline{x} ; \alpha, \beta)}{\partial \beta^{2}}=\sum_{i=1}^{m} \frac{1}{\left(\beta+x_{i}^{2}\right)^{2}}+\frac{\alpha}{\beta^{2}} \sum_{i=1}^{m} x_{i}^{2}\left(R_{i}+1\right)\left[\frac{x_{i}^{2} / \beta}{\left(1+\left(x_{i}^{2} / \beta\right)\right)^{2}}-\frac{2}{1+\left(x_{i}^{2} / \beta\right)}\right] .
\end{gathered}
$$

The asymptotic normality of the MLE can be used to compute the approximate confidence intervals for parameters $\alpha$, and $\beta$. Therefore, $(1-\gamma) 100 \%$ confidence intervals for parameters $\alpha$, and $\beta$ become

$$
\widehat{\alpha} \pm Z_{\gamma / 2} \sqrt{\operatorname{var}(\widehat{\alpha})} \quad \text { and } \quad \widehat{\beta} \pm Z_{\gamma / 2} \sqrt{\operatorname{var}(\widehat{\beta})}
$$

where $Z_{\gamma / 2}$ is the percentile of the standard normal distribution with right-tail probability $\gamma / 2$.

\section{Parametric bootstrap confidence intervals}

A parametric bootstrap interval provides much more information about the population value of the quantity of interest than does a point estimate. In this section, we propose to use confidence intervals based on the parameteric bootstrap methods (i) percentile bootstrap method (Boot-p) based on the idea of Efron [10]. (ii) bootstrap-t method (Boot-t) based on the idea of Hall [14]. The algorithms for estimating the confidenc eintervals using both methods are illustrated as follows

\subsection{Percentile bootstrap method (Boot-p)}

\section{Algorithm 1.}


Step 1 From the original data $\underline{x} \equiv x_{1: m: n}^{\mathbf{R}}, x_{2: m: n}^{\mathbf{R}}, \ldots, x_{m: m: n}^{\mathbf{R}}$ compute the ML estimates of the parameters $\widehat{\alpha}$ and $\widehat{\beta}$ by solving the equations (2.5) and (2.6).

Step 2 Use $\widehat{\alpha}$ and $\widehat{\beta}$ to generate a bootstrap sample $\underline{x}^{*}$ with the same values of $R_{i}, m ;(i=1,2, . ., m)$ using algorithm presented in [6].

Step 3 As in Step 1, based on $x^{*}$ compute the bootstrap sample estimates of $\alpha$ and $\beta$, say $\widehat{\alpha}^{*}$ and $\widehat{\beta}^{*}$.

Stap 4 Repeat Steps 2-3 $B$ times representing $B$ bootstrap MLE's of $\alpha$, and $\beta$ based on $B$ different bootstrap samples.

Step 5 Arrange all $\widehat{\alpha}^{* \prime} s$ and $\widehat{\beta}^{* \prime} s$, in an ascending order to obtain the bootstrap sample $\left(\varphi_{l}^{[1]}, \varphi_{l}^{[2]}\right.$, $\left.\ldots, \varphi_{l}^{[B]}\right), l=1,2\left(\right.$ where $\left.\varphi_{1} \equiv \widehat{\alpha}^{*}, \varphi_{2} \equiv \widehat{\beta}^{*}\right)$.

Let $G(z)=P\left(\varphi_{l} \leq z\right)$ be the cumulative distribution function of $\varphi_{1}$. Define $\varphi_{\text {lboot }}=G^{-1}(z)$ for given $z$. The approximate bootstrap $100(1-2 \gamma) \%$ confidence interval of $\varphi_{l}$ is given by

$$
\left[\varphi_{\text {lboot }}(\gamma), \varphi_{\text {lboot }}(1-\gamma)\right] \text {. }
$$

\subsection{Bootstrap-t method (Boot-t)}

\section{Algorithm 2.}

Step 1 From the original data $\underline{x} \equiv x_{1: m: n}^{\mathbf{R}}, x_{2: m: n}^{\mathbf{R}}, \ldots, x_{m: m: n}^{\mathbf{R}}$ compute the ML estimates of the parameters $\widehat{\alpha}$ and $\widehat{\beta}$ by solving the Eqs. (2.5) and (2.6).

Step 2 Using $\widehat{\alpha}$ and $\widehat{\beta}$ generate abootstrap sample $\underline{x}^{*}$. Based on $\underline{x}^{*}$ compute the bootstrap estimate of $\alpha$ and $\beta$ using (2.5) and (2.6), say $\widehat{\alpha}^{*}$ and $\widehat{\beta}^{*}$ and the following statistics

$$
T_{1}^{*}=\frac{\sqrt{B}\left(\widehat{\alpha}^{*}-\widehat{\alpha}\right)}{\sqrt{\operatorname{Var}\left(\widehat{\alpha}^{*}\right)}} \text { and } T_{2}^{*}=\frac{\sqrt{B}\left(\widehat{\beta}^{*}-\widehat{\beta}\right)}{\sqrt{\operatorname{Var}\left(\widehat{\beta}^{*}\right)}}
$$

where $\operatorname{Var}\left(\widehat{\alpha}^{*}\right)$ and $\operatorname{Var}\left(\widehat{\beta}^{*}\right)$ are obtained using the Fisher information matrix.

Step 3 Repeat Step 2, B boot times.

Stap 4 For the $T_{1}^{*}$ and $T_{2}^{*}$ values obtained in Step2, determine the upper and lower bounds of the $100(1-\gamma) \%$ confidence interval of $\alpha$ and $\beta$ as follows: let $H(x)=P\left(T_{i}^{*} \leq x\right), i=1,2$ be the cumulative distribution function of $T_{1}^{*}$ and $T_{2}^{*}$. For a given $x$, define

$$
\widehat{\alpha}_{B o o t-t}(x)=\widehat{\alpha}+B^{-1 / 2} \sqrt{\operatorname{Var}(\widehat{\alpha})} H^{-1}(x) \text { and } \widehat{\beta}_{\text {Boot-t }}(x)=\widehat{\beta}+B^{-1 / 2} \sqrt{\operatorname{Var}(\widehat{\beta})} H^{-1}(x) .
$$

Here also, $\operatorname{Var}(\widehat{\alpha})$ and $\operatorname{Var}(\widehat{\beta})$ can be computed as same as computing the $\operatorname{Var}\left(\widehat{\alpha}^{*}\right)$ and $\operatorname{Var}\left(\widehat{\beta}^{*}\right)$. The approximate $100(1-2 \gamma) \%$ confidence interval of $\alpha$ and $\beta$ are given by

$$
\left(\widehat{\alpha}_{\text {Boot }-t}(\gamma), \widehat{\alpha}_{\text {Boot }-t}(1-\gamma)\right) \text { and }\left(\widehat{\beta}_{\text {Boot }-t}(\gamma), \widehat{\beta}_{\text {Boot-t }}(1-\gamma)\right) \text {. }
$$

\section{Bayesian estimation}

In this section we describe how to obtain the Bayes estimates and the corresponding credible intervals of parameters $\alpha$ and $\beta$ when both are unknown. For computing the Bayes estimates, we assume 
mainly a squared error loss (SEL) function. Now let us consider independent gamma priors for the parameters $\alpha$ and $\beta$ with the pdfs as

$$
\begin{aligned}
& \pi_{1}(\alpha \mid a, b) \propto \alpha^{a-1} e^{-b \alpha}, \quad \alpha>0, \quad a>0, \quad b>0 \\
& \pi_{2}(\beta \mid c, d) \propto \beta^{c-1} e^{-d \beta}, \quad \beta>0, \quad c>0, \quad d>0
\end{aligned}
$$

where $a, b, c$ and $d$ are assumed to be known and are chosen to reflect prior knowledge about $\alpha$ and $\beta$. Note that when $a=b=c=d=0$, (we call it prior 0) they are the non-informative priors of $\alpha$ and $\beta$, respectively.

It follows from (2.3), (4.1) and (4.2) that the joint posterior density function of $\alpha$ and $\beta$ given $\underline{x}$ is thus

$$
\begin{aligned}
\pi^{*}(\alpha, \beta \mid \underline{x})= & \frac{L(\underline{x} ; \alpha, \beta) \times \pi_{1}(\alpha \mid a, b) \times \pi_{2}(\beta \mid c, d)}{\int_{0}^{\infty} \int_{0}^{\infty} L(\underline{x} ; \alpha, \beta) \times \pi_{1}(\alpha \mid a, b) \times \pi_{2}(\beta \mid c, d) d \alpha d \beta} \\
\propto & \alpha^{m+a-1} \beta^{c-1} \exp \left[-\alpha \sum_{i=1}^{m}\left(R_{i}+1\right) \log \left(1+\frac{x_{i}^{2}}{\beta}\right)-d \beta\right] \\
& \times\left(\prod_{i=1}^{m} \frac{x_{i}}{\left(\beta+x_{i}^{2}\right)}\right)
\end{aligned}
$$

It is not possible to compute (4.3) analytically. The problem is that the integrals in (4.3) are usually impossible to evaluate analytically, and the numerical methods may fail. The MCMC method provides an alternative method for parameter estimation. In the following subsections, we propose using the MCMC technique to obtain Bayes estimates of the unknown parameters and construct the corresponding credible intervals.

\subsection{MCMC technique}

Markov chain Monte Carlo (MCMC) methods use computer simulation of Markov chains in the parameter space Gilks et al. [11]. The Markov chains are defined in such a way that the posterior distribution in the given statistical inference problem is the asymptotic distribution. This allows to use ergodic averages to approximate the desired posterior expectations. Several standard approaches to define such Markov chains exist, including Gibbs sampling, Metropolis-Hastings $(\mathrm{MH})$ and reversible jump. The MH algorithm is a very general MCMC method first developed by Metropolis et al. [19] and later extended by Hastings [15]. Using these algorithms it is possible to implement posterior simulation in essentially any problem which allow pointwise evaluation of the prior distribution and likelihood function. It can be used to obtain random samples from any arbitrarily complicated target distribution of any dimension that is known up to a normalizing constant. In fact, Gibbs sampler is just a special case of the MH algorithm. Details of the MCMC method can be found in $[11,21]$.

In order to use the method of MCMC for estimating the parameters of the $\mathrm{CR}(\alpha, \beta)$ distribution, namely, $\alpha$ and $\beta$. Let us consider independent priors as in (4.1) and (4.2), the full conditional distribution for any parameter can be obtained, to within a constant, by factoring out from the likelihood function $L(\underline{x} ; \alpha, \beta)$ any terms containing the relevant parameter and multiplying by its 
prior. From (4.3), the marginal posterior density of $\alpha$ is proportional to

$$
\pi_{1}^{*}(\alpha \mid \beta, \underline{x}) \propto \alpha^{m+a-1} \exp \left[-\alpha\left(b+\sum_{i=1}^{m}\left(R_{i}+1\right) \log \left(1+\frac{x_{i}^{2}}{\beta}\right)\right)\right] .
$$

Similarly, the full posterior conditional distribution for $\beta$ is proportional to

$$
\pi_{2}^{*}(\beta \mid \alpha, \underline{x}) \propto \beta^{c-1} \exp \left[-\alpha \sum_{i=1}^{m}\left(R_{i}+1\right) \log \left(1+\frac{x_{i}^{2}}{\beta}\right)-d \beta\right] \times\left(\prod_{i=1}^{m} \frac{x_{i}}{\left(\beta+x_{i}^{2}\right)}\right) .
$$

It can be seen that Eq. (4.4) is a gamma density with shape parameter $(m+a)$ and scale parameter $b+\sum_{i=1}^{m}\left(R_{i}+1\right) \log \left(1+\frac{x_{i}^{2}}{\beta}\right)$ and, therefore, samples of $\alpha$ can be easily generated using any gamma generating routine. But the conditional posterior distribution of $\beta$ Eq. (4.5) cannot be reduced analytically to well known distributions and therefore it is not possible to sample directly by standard methods, but the plot of it show that it is similar to normal distribution. So to generate random numbers from this distribution, we use the Metropolis-Hastings method with normal proposal distribution.

Now, we propose the following scheme to generate $\alpha$ and $\beta$ from the posterior density functions and in turn obtain the Bayes estimates and the corresponding credible intervals

Step 1 Start with an $\beta^{(0)}=\hat{\beta}, M=$ burn - in.

Step 2 Set $t=1$.

Step 3 Generate $\alpha^{(t)}$ from Gamma distribution $\pi_{1}^{*}(\alpha \mid \beta, \underline{x})$.

Step 4 Using Metropolis-Hastings (see, Metropolis et al. [19]), generate $\beta^{(t)}$ from $\pi_{2}^{*}(\beta \mid \alpha, \underline{x})$ with the $\mathrm{N}\left(\beta^{(t-1)}, \sigma^{2}\right)$ proposal distribution where $\sigma^{2}$ is the variance of $\beta$ obtained using variance-covariance matrix.

Step 5 Compute $\beta^{(t)}$ and $\alpha^{(t)}$.

Step 6 Set $t=t+1$.

Step 7 Repeat Steps 3-6 N times.

Step 8 Obtain the Bayes estimates of $\beta$ and $\alpha$ with respect to the SEL function as

$$
\begin{aligned}
& \widehat{E}(\beta \mid \underline{x})=\frac{1}{N-M} \sum_{i=M+1}^{N} \beta_{i}, \quad \text { and } \\
& \widehat{E}(\alpha \mid \underline{x})=\frac{1}{N-M} \sum_{i=M+1}^{N} \alpha_{i} .
\end{aligned}
$$

Step 9 To compute the credible intervals of $\beta$ and $\alpha$, order $\beta_{1}, \ldots, \beta_{N-M}$ and $\alpha_{1}, \ldots, \alpha_{N-M}$ as $\beta_{(1)}<$ $\ldots<\beta_{(N-M)}$ and $\alpha_{(1)}<\ldots<\alpha_{(N-M)}$. Then the $100(1-\gamma) \%$ symmetric credible intervals of $\beta$ and $\alpha$ become

$$
\left(\beta_{((N-M) \gamma / 2)}, \beta_{((N-M)(1-\gamma / 2))}\right) \text { and }\left(\alpha_{((N-M) \gamma / 2)}, \alpha_{((N-M)(1-\gamma / 2))}\right) .
$$

\section{Illustrative example (real data)}

To illustrate the application of our proposed method, we chose the real data set which was reported by [9] and represents the survival times in years of a group of patients given chemotherapy treatment. The data consisting of 46 survival times (in years) for 46 patients are: 0.047, 0.115, 0.121, 
$0.132,0.164,0.197,0.203,0.260,0.282,0.296,0.334,0.395,0.458,0.466,0.501,0.507,0.529$, $0.534,0.540,0.570,0.641,0.644,0.696,0.841,0.863,1.099,1.219,1.271,1.326,1.447,1.485$, $1.553,1.581,1.589,2.178,2.343,2.416,2.444,2.825,2.830,3.578,3.658,3.743,3.978,4.003$, 4.033. Reference [9] shows that the Compound Rayleigh model is acceptable for these data. then we have a previous data consisting of 46 survival times from the Compound Rayleigh distribution (CRD), using the algorithm described in Balakrishnan and Sandhu [6], we generate a progressively type-II censored sample of size 26 out of 46 is obtained as $\left(X_{1}, \ldots X_{26}\right)=0.047,0.132,0.203,0.260$, $0.282,0.395,0.501,0.570,0.641,0.696,0.841,0.863,1.099,1.219,1.271,1.326,1.447,1.553$, $1.581,1.589,2.178,2.416,2.444,2.825,4.003,4.033$. In this case $n=46, m=26$ and the censoring scheme $R=\left(R_{1}=20, R_{2}=R_{3}=\cdots=R_{26}=0\right)$, for simplicity we denoted to this censoring scheme (C.S) by $\left(20,25^{\circ}\right)$. For this example, 20 patient's survival times are censored, and 26 times are observed.

Based on these data, we compute the approximate MLEs, Bootstrap confidence intervals (Boot$\mathrm{p}$, Boot-t) and Bayes estimates of $\alpha$ and $\beta$ using MCMC method, we assume that informative priors $a=2, b=2, c=2$ and $d=2)$ on both $\alpha$ and $\beta$.The density function of $\pi_{2}^{*}(\beta \mid \alpha, \underline{x})$ as given in (4.5) is plotted in Figure 1. It can be approximated by normal distribution function as mentioned in Subsection 4.1. Also, we compute the 95\%, approximate confidence intervals (ACI), Bootstrap confidence intervals and approximate credible intervals based on the MCMC samples. The results are given in Table 1. Figures 2 and 3 plot the MCMC out put of $\alpha$ and $\beta$, using 10000 MCMC samples (dashed line represent means and red lines represent lower and upper bounds of 95\% probability intervals.). The plot of histogram of $\alpha$ and $\beta$ generated by MCMC method are given in Figures 4 and 5. This was done with 1000 bootstrap sample and 10000 MCMC sample and discard the first 1000 values as 'burn-in'.

Table 1. Results obtained by MLEs, Bootstrap and MCMC method of $\alpha$ and $\beta$.

\begin{tabular}{ccccc}
\hline Method & Parameter & Point & Interval & Length \\
\hline MLEs & $\alpha$ & 1.1463 & {$[-0.0501,2.3428]$} & 2.3928 \\
& $\beta$ & 1.1820 & {$[-0.9624,3.3265]$} & 4.2888 \\
Bootstrap-p & $\alpha$ & 1.1504 & {$[0.6350,3.0612]$} & 2.4262 \\
& $\beta$ & 1.1917 & {$[0.4511,4.2148]$} & 3.7637 \\
Bootstrap-t & $\alpha$ & 1.1514 & {$[0.1791,2.6884]$} & 2.5094 \\
& $\beta$ & 1.1873 & {$[0.1329,4.1615]$} & 4.0286 \\
Bayes (MCMC) & $\alpha$ & 1.1058 & {$[0.5743,1.8778]$} & 1.3035 \\
& $\beta$ & 1.1685 & {$[0.3401,2.5667]$} & 2.2266 \\
\hline
\end{tabular}




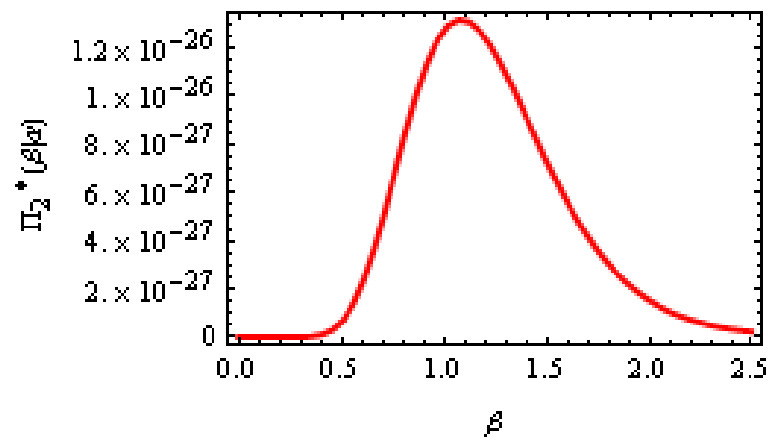

Fig. 1. Posterior density function of $\beta$ given $\alpha$

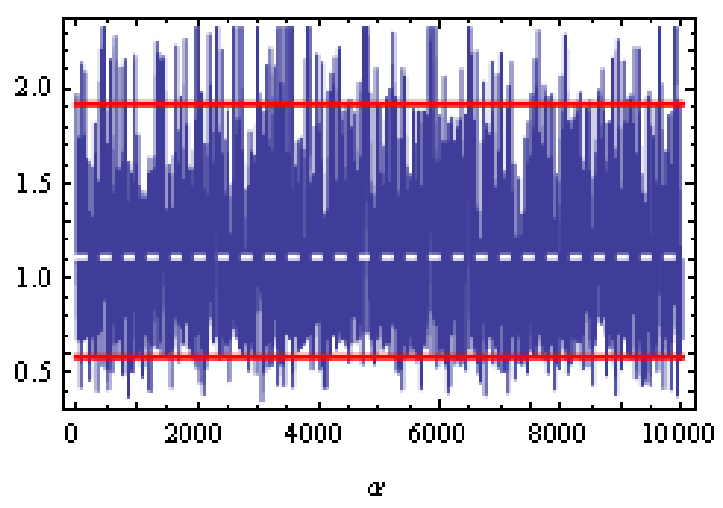

Fig. 2. Simulation number of $\alpha$ generated by MCMC method.

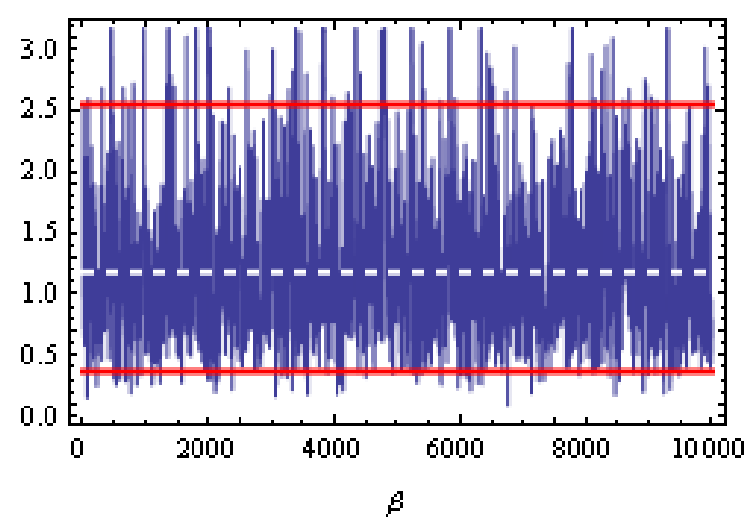

Fig. 3. Simulation number of $\beta$ generated by MCMC method. 


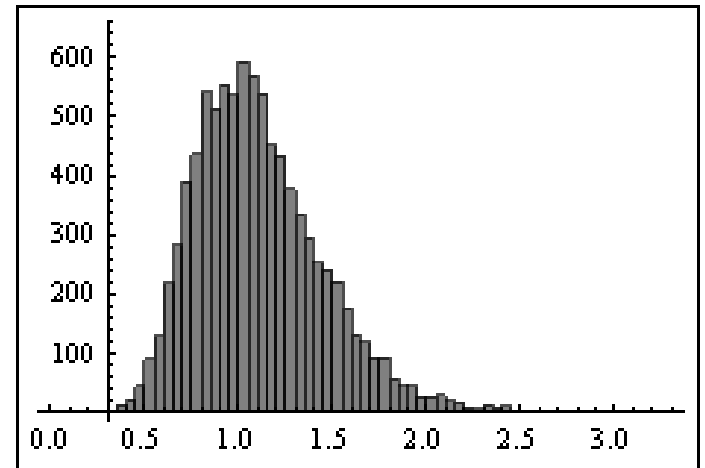

Fig. 4. Histogram of $\alpha$ generated by MCMC method.

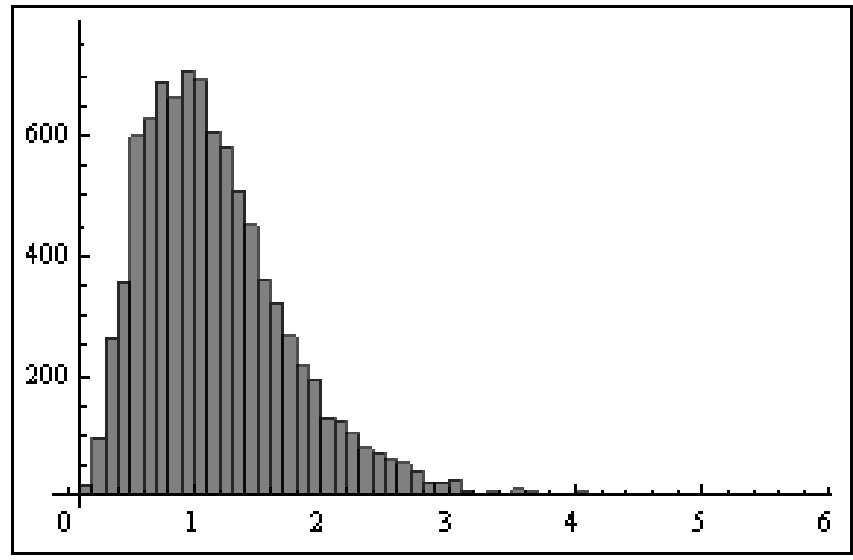

Fig. 5. Histogram of $\beta$ generated by MCMC method.

\section{Simulation study}

In order to compare the parameters estimators, Monte Carlo simulations were performed utilizing 1000 progressively type-II censored samples for each simulations. The mean square error (MSE) is used to compare the estimators. The samples were generated by using the algorithm described in Balakrishnan and Sandhu [6] using $(\alpha, \beta)=(1,2),(0.5,1)$ with different sample of sizes $(n)$, different effective sample of sizes $(m)$ and different of sampling schemes (i.e., different $R_{i}$ values). Also, we used different hyperparameters $a, b, c$ and $d$. First, we used the noninformative gamma priors for both the parameters, that is, when the hyperparameters are 0. We call it prior 0: $a=b=c=d=0$. Note that as the hyperparameters go to 0 , the prior density becomes inversely proportional to its argument and also becomes improper. This density is commonly used as an improper prior for parameters in the range of 0 to infinity, and this prior is not specifically related to the gamma density. For computing Bayes estimators, other than prior 0, we also used informative prior, including prior $1, a=1, b=2, c=2$ and $d=1$. In two cases, we used the squared error loss function to compute the Bayes estimates. We also computed the Bayes estimates and 95\% credible intervals based on 10000 MCMC samples and discard the first 1000 values as 'burn-in'. We report the average 
Bayes estimates, mean squared errors MSEs, coverage percentages, and average confidence interval lengths. For comparison purposes, we also compute the MLEs and the 95\% confidence intervals based on the observed Fisher information matrix. Finally, we used the same 1000 replicates to compute different estimates for each scheme Tables 2-5 report the results based on MLEs and the Bayes estimators using noninformative prior and informative prior on both $\alpha$ and $\beta$.

Table 2. Average values of the different estimators and the corresponding MSEs when $\alpha=1$ and $\beta=2$.

\begin{tabular}{|c|c|c|c|c|c|c|c|c|}
\hline \multirow[t]{2}{*}{$\mathrm{n}$} & \multirow[t]{2}{*}{$\mathrm{m}$} & \multirow[t]{2}{*}{ Scheme } & \multicolumn{2}{|c|}{ MLE } & \multicolumn{2}{|c|}{ (MCMC) (Prior 0) } & \multicolumn{2}{|c|}{ (MCMC)(Prior 1) } \\
\hline & & & $\alpha$ & $\beta$ & $\alpha$ & $\beta$ & $\alpha$ & $\beta$ \\
\hline \multirow[t]{6}{*}{30} & \multirow[t]{6}{*}{20} & \multirow[t]{2}{*}{$\left(10,19^{0}\right)$} & 1.2420 & 2.007 & 1.2644 & 2.0624 & 0.9414 & 2.1117 \\
\hline & & & $(0.0459)$ & $(0.3640)$ & $(0.0462)$ & $(0.3940)$ & $(0.0355)$ & $(0.2030)$ \\
\hline & & \multirow[t]{2}{*}{$\left(5^{0}, 10^{1}, 5^{0}\right)$} & 1.2085 & 2.1498 & 1.2414 & 2.2049 & 0.9882 & 2.0871 \\
\hline & & & $(0.0586)$ & $(0.4338)$ & $(0.0599)$ & $(0.4425)$ & $(0.0378)$ & $(0.2341)$ \\
\hline & & \multirow[t]{2}{*}{$\left(19^{0}, 10\right)$} & 1.1822 & 2.1112 & 1.9331 & 2.1906 & 0.9977 & 2.0098 \\
\hline & & & $(0.0593)$ & $(0.5320)$ & $(0.0618)$ & $(0.5328)$ & $(0.0461)$ & $(0.2769)$ \\
\hline \multirow[t]{6}{*}{40} & \multirow[t]{6}{*}{20} & \multirow[t]{2}{*}{$\left(20,19^{0}\right)$} & 1.0356 & 2.0321 & 1.0468 & 2.1837 & 0.9682 & 2.1004 \\
\hline & & & $(0.0465)$ & $(0.3849)$ & $(0.0478)$ & $(0.4012)$ & $(0.0341)$ & $(0.2135)$ \\
\hline & & \multirow[t]{2}{*}{$\left(20^{1}\right)$} & 1.0043 & 2.1226 & 1.0776 & 2.1228 & 0.9912 & 2.0004 \\
\hline & & & $(0.0596)$ & $(0.4403)$ & $(0.0597)$ & $(0.4597)$ & $(0.0360)$ & $(0.2388)$ \\
\hline & & \multirow[t]{2}{*}{$\left(19^{0}, 20\right)$} & 1.1175 & 2.1917 & 1.2081 & 2.1278 & 0.9867 & 1.9982 \\
\hline & & & $(0.0613)$ & $(0.5382)$ & $(0.0623)$ & $(0.5471)$ & $(0.0466)$ & $(0.2870)$ \\
\hline \multirow[t]{6}{*}{40} & \multirow[t]{6}{*}{30} & \multirow[t]{2}{*}{$\left(10,29^{0}\right)$} & 1.1978 & 2.1795 & 1.1547 & 2.1833 & 0.9769 & 2.1578 \\
\hline & & & $(0.0379)$ & $(0.3536)$ & $(0.0385)$ & $(0.3692)$ & $(0.0295)$ & $(0.1992)$ \\
\hline & & \multirow[t]{2}{*}{$\left(10^{0}, 10^{1}, 10^{0}\right)$} & 1.2295 & 2.0222 & 1.0407 & 2.0640 & 0.9971 & 2.0358 \\
\hline & & & $(0.0426)$ & $(0.3882)$ & $(0.0429)$ & $(0.3907)$ & $(0.0337)$ & $(0.2097)$ \\
\hline & & \multirow[t]{2}{*}{$\left(29^{0}, 10\right)$} & 1.1337 & 2.1644 & 1.1025 & 2.1283 & 0.9382 & 2.0817 \\
\hline & & & $(0.0507)$ & $(0.4781)$ & $(0.0511)$ & (0.4799) & $(0.0418)$ & $(0.2574)$ \\
\hline \multirow[t]{6}{*}{50} & \multirow[t]{6}{*}{40} & \multirow[t]{2}{*}{$\left(10,39^{0}\right)$} & 1.1636 & 2.1917 & 1.1899 & 2.1401 & 0.9876 & 2.1515 \\
\hline & & & $(0.0276)$ & $(0.3141)$ & $(0.0278)$ & $(0.3220)$ & $(0.0223)$ & $(0.1522)$ \\
\hline & & \multirow[t]{2}{*}{$\left(15^{0}, 10^{1}, 15^{0}\right)$} & 1.1678 & 2.1899 & 1.1191 & 2.0777 & 0.9814 & 2.0076 \\
\hline & & & $(0.0290)$ & $(0.3328)$ & $(0.0294)$ & $(0.3405)$ & $(0.0244)$ & $(0.1712)$ \\
\hline & & \multirow[t]{2}{*}{$\left(39^{0}, 10\right)$} & 1.1535 & 2.0078 & 1.0636 & 2.1459 & 0.9786 & 2.0214 \\
\hline & & & $(0.0317)$ & $(0.3597)$ & $(0.0318)$ & $(0.3603)$ & $(0.0266)$ & $(0.2102)$ \\
\hline
\end{tabular}

Note: Corresponding to each scheme, the first figure represents the average estimates, with the corresponding MSEs reported below it in parentheses. 
Table 3. Average confidence interval, credible interval lengths and the coverage percentages when $\alpha=1$ and $\beta=2$.

\begin{tabular}{|c|c|c|c|c|c|c|c|c|}
\hline \multirow[t]{2}{*}{$\mathrm{n}$} & \multirow[t]{2}{*}{$\mathrm{m}$} & \multirow[t]{2}{*}{ Scheme } & \multicolumn{2}{|c|}{ MLE } & \multicolumn{2}{|c|}{ (MCMC) (Prior 0) } & \multicolumn{2}{|c|}{ (MCMC)(Prior 1) } \\
\hline & & & $\alpha$ & $\beta$ & $\alpha$ & $\beta$ & $\alpha$ & $\beta$ \\
\hline \multirow[t]{6}{*}{30} & \multirow[t]{6}{*}{20} & $\left(10,19^{0}\right)$ & 2.6570 & 4.0966 & 2.6935 & 4.6438 & 1.1577 & 3.9058 \\
\hline & & & $(0.941)$ & $(0.945)$ & $(0.945)$ & $(0.947)$ & $(0.955)$ & $(0.945)$ \\
\hline & & $\left(5^{0}, 10^{1}, 5^{0}\right)$ & 2.7250 & 4.1689 & 2.7253 & 4.6949 & 1.2211 & 3.9374 \\
\hline & & & $(0.952)$ & $(0.946)$ & $(0.942)$ & $(0.939)$ & $(0.970)$ & $(0.961)$ \\
\hline & & $\left(19^{0}, 10\right)$ & 2.7505 & 4.2222 & 2.9723 & 4.7151 & 1.2978 & 3.9455 \\
\hline & & & $(0.965)$ & $(0.954)$ & $(0.949)$ & $(0.953)$ & $(0.966)$ & $(0.962)$ \\
\hline \multirow[t]{6}{*}{40} & \multirow[t]{6}{*}{20} & $\left(20,19^{0}\right)$ & 2.6925 & 4.1017 & 2.7260 & 4.6702 & 1.1914 & 3.9348 \\
\hline & & & $(0.949)$ & $(0.947)$ & $(0.954)$ & $(0.945)$ & $(0.955)$ & $(0.963)$ \\
\hline & & $\left(20^{1}\right)$ & 2.7322 & 4.1763 & 2.7398 & 4.6974 & 1.2671 & 3.9482 \\
\hline & & & $(0.960)$ & $(0.948)$ & $(0.962)$ & $(0.953)$ & $(0.954)$ & $(0.966)$ \\
\hline & & $\left(19^{0}, 20\right)$ & 2.7569 & 4.2583 & 2.7989 & 4.7194 & 1.3035 & 3.9818 \\
\hline & & & $(0.952)$ & $(0.956)$ & $(0.949)$ & $(0.951)$ & $(0.944)$ & $(0.946)$ \\
\hline \multirow[t]{6}{*}{40} & \multirow[t]{6}{*}{30} & $\left(10,29^{0}\right)$ & 2.3322 & 3.9277 & 2.6123 & 4.3452 & 1.1297 & 3.6298 \\
\hline & & & $(0.947)$ & $(0.948)$ & $(0.961)$ & $(0.959)$ & $(0.956)$ & $(0.945)$ \\
\hline & & $\left(10^{0}, 10^{1}, 10^{0}\right)$ & 2.4651 & 3.9658 & 2.7096 & 4.3701 & 1.1984 & 3.8698 \\
\hline & & & $(0.957)$ & $(0.948)$ & $(0.965)$ & $(0.943)$ & $(0.945)$ & $(0.947)$ \\
\hline & & $\left(29^{0}, 10\right)$ & 2.6376 & 4.1062 & 2.7326 & 4.4306 & 1.2034 & 3.8863 \\
\hline & & & $(0.945)$ & $(0.953)$ & $(0.941)$ & $(0.939)$ & $(0.955)$ & $(0.942)$ \\
\hline \multirow[t]{6}{*}{50} & \multirow[t]{6}{*}{40} & $\left(10,39^{0}\right)$ & 1.7733 & 3.5792 & 1.8388 & 3.9629 & 1.0968 & 3.3384 \\
\hline & & & $(0.950)$ & $(0.943)$ & $(0.949)$ & $(0.946)$ & $(0.964)$ & $(0.941)$ \\
\hline & & $\left(15^{0}, 10^{1}, 15^{0}\right)$ & 1.8724 & 3.6093 & 2.0194 & 4.0239 & 1.1158 & 3.3782 \\
\hline & & & $(0.955)$ & $(0.940)$ & $(0.941)$ & $(0.939)$ & $(0.945)$ & $(0.935)$ \\
\hline & & $\left(39^{0}, 10\right)$ & 2.1281 & 3.7378 & 2.3771 & 4.1756 & 1.1316 & 3.5862 \\
\hline & & & $(0.953)$ & $(0.941)$ & $(0.954)$ & $(0.940)$ & $(0.951)$ & $(0.939)$ \\
\hline
\end{tabular}

Note: Corresponding to each scheme, the first figure represents the average confidence interval and credible interval lengths, with the corresponding coverage percentage reported below it in parentheses. 
Table 4. Average values of the different estimators and the corresponding MSEs when $\alpha=0.5$ and $\beta=1$

\begin{tabular}{|c|c|c|c|c|c|c|c|c|}
\hline \multirow[t]{2}{*}{$\mathrm{n}$} & \multirow[t]{2}{*}{$\overline{\mathrm{m}}$} & \multirow[t]{2}{*}{ Scheme } & \multicolumn{2}{|c|}{ MLE } & \multicolumn{2}{|c|}{ 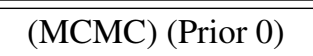 } & \multicolumn{2}{|c|}{ 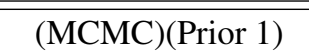 } \\
\hline & & & $\alpha$ & $\beta$ & $\alpha$ & $\beta$ & $\alpha$ & $\beta$ \\
\hline \multirow[t]{6}{*}{30} & 20 & $\left(10,19^{0}\right)$ & 0.6137 & 1.1170 & 0.6248 & 1.1350 & 0.6109 & 1.0425 \\
\hline & & & $(0.0092)$ & $(0.0350)$ & $(0.0108)$ & $(0.0371)$ & $(0.0088)$ & $(0.0297)$ \\
\hline & & $\left(5^{0}, 10^{1}, 5^{0}\right)$ & 0.6103 & 1.1773 & 0.6245 & 1.1698 & 0.6085 & 1.0135 \\
\hline & & & $(0.0098)$ & $(0.0374)$ & $(0.0113)$ & $(0.0388)$ & $(0.0093)$ & $(0.0310)$ \\
\hline & & $\left(19^{0}, 10\right)$ & 0.6065 & 0.9754 & 0.5434 & 1.1036 & 0.5307 & 0.9834 \\
\hline & & & $(0.0103)$ & $(0.0409)$ & $(0.0116)$ & $(0.0411)$ & $(0.0098)$ & $(0.0337)$ \\
\hline \multirow[t]{6}{*}{40} & 20 & $\left(20,19^{0}\right)$ & 0.5719 & 1.1641 & 0.6150 & 1.1485 & 0.5157 & 1.0657 \\
\hline & & & $(0.0093)$ & $(0.0361)$ & $(0.0110)$ & $(0.0382)$ & $(0.0091)$ & $(0.0299)$ \\
\hline & & $\left(20^{1}\right)$ & 0.5038 & 1.0770 & 0.5356 & 1.0802 & 0.5028 & 1.0528 \\
\hline & & & $(0.0100)$ & $(0.0375)$ & $(0.0114)$ & $(0.0391)$ & $(0.0096)$ & $(0.0316)$ \\
\hline & & $\left(19^{0}, 20\right)$ & 0.5121 & 1.1720 & 0.5861 & 1.1736 & 0.5107 & 0.9866 \\
\hline & & & $(0.0114)$ & $(0.0413)$ & $(0.0123)$ & $(0.0427)$ & $(0.0102)$ & $(0.0353)$ \\
\hline \multirow[t]{6}{*}{40} & 30 & $\left(10,29^{0}\right)$ & 0.5967 & 1.1091 & 0.6102 & 1.1576 & 0.6047 & 1.0827 \\
\hline & & & $(0.0087)$ & $(0.0298)$ & $(0.0098)$ & $(0.0304)$ & $(0.0082)$ & $(0.0275)$ \\
\hline & & $\left(10^{0}, 10^{1}, 10^{0}\right)$ & 0.5298 & 1.1067 & 0.5764 & 1.1774 & 0.5189 & 1.0494 \\
\hline & & & $(0.0095)$ & $(0.0311)$ & $(0.0103)$ & $(0.0314)$ & $(0.0089)$ & $(0.0287)$ \\
\hline & & $\left(29^{0}, 10\right)$ & 0.5968 & 1.15817 & 0.5424 & 1.1744 & 0.5137 & 1.0091 \\
\hline & & & $(0.0102)$ & $(0.0346)$ & $(0.0107)$ & $(0.0351)$ & $(0.0098)$ & $(0.0312)$ \\
\hline \multirow[t]{6}{*}{50} & 40 & $\left(10,39^{0}\right)$ & 0.5441 & 1.1539 & 0.5642 & 1.1694 & 0.5245 & 1.1132 \\
\hline & & & $(0.0079)$ & $(0.0215)$ & $(0.0083)$ & $(0.0229)$ & $(0.0073)$ & $(0.0211)$ \\
\hline & & $\left(15^{0}, 10^{1}, 15^{0}\right)$ & 0.5250 & 1.2204 & 0.5542 & 1.2168 & 0.5150 & 1.1525 \\
\hline & & & $(0.0083)$ & $(0.0221)$ & $(0.086)$ & $(0.0259)$ & $(0.0081)$ & $(0.0222)$ \\
\hline & & $\left(39^{0}, 10\right)$ & 0.5886 & 1.1818 & 0.6078 & 1.0903 & 0.6085 & 1.0499 \\
\hline & & & $(0.0097)$ & $(0.0248)$ & $(0.0098)$ & $(0.0261)$ & $(0.0087)$ & $(0.0214)$ \\
\hline
\end{tabular}

Note: Corresponding to each scheme, the first figure represents the average estimates, with the corresponding MSEs reported below it in parentheses. 
Table 5. Average confidence interval, credible interval lengths and the coverage percentages when $\alpha=0.5$ and $\beta=1$.

\begin{tabular}{|c|c|c|c|c|c|c|c|c|}
\hline \multirow[t]{2}{*}{$\mathrm{n}$} & \multirow[t]{2}{*}{$\mathrm{m}$} & \multirow[t]{2}{*}{ Scheme } & \multicolumn{2}{|c|}{ MLE } & \multicolumn{2}{|c|}{ (MCMC) (Prior 0) } & \multicolumn{2}{|c|}{ (MCMC)(Prior 1) } \\
\hline & & & $\alpha$ & $\beta$ & $\alpha$ & $\beta$ & $\alpha$ & $\beta$ \\
\hline \multirow[t]{6}{*}{30} & 20 & $\left(10,19^{0}\right)$ & 1.0174 & 2.3867 & 1.0197 & 2.0963 & 1.0155 & 2.0884 \\
\hline & & & $(0.955)$ & $(0.949)$ & $(0.947)$ & $(0.943)$ & $(0.956)$ & $(0.945)$ \\
\hline & & $\left(5^{0}, 10^{1}, 5^{0}\right)$ & 1.1194 & 2.4151 & 1.1136 & 2.4171 & 1.0167 & 2.0945 \\
\hline & & & $(0.954)$ & $(0.942)$ & $(0.961)$ & $(0.939)$ & $(0.956)$ & $(0.947)$ \\
\hline & & $\left(19^{0}, 10\right)$ & 1.1750 & 2.6012 & 1.2104 & 2.6348 & 1.0221 & 2.1279 \\
\hline & & & $(0.945)$ & $(0.937)$ & $(0.936)$ & $(0.941)$ & $(0.951)$ & $(0.944)$ \\
\hline \multirow[t]{6}{*}{40} & 20 & $\left(20,19^{0}\right)$ & 1.0181 & 2.3961 & 1.0201 & 2.0987 & 1.0162 & 2.0893 \\
\hline & & & $(0.954)$ & $(0.937)$ & $(0.961)$ & $(0.948)$ & $(0.952)$ & $(0.949)$ \\
\hline & & $\left(20^{1}\right)$ & 1.0197 & 2.4157 & 1.0216 & 2.4195 & 1.0185 & 2.0959 \\
\hline & & & $(0.953)$ & (0.949) & $(0.944)$ & $(0.937)$ & $(0.945)$ & $(0.936)$ \\
\hline & & $\left(19^{0}, 20\right)$ & 1.2195 & 2.8294 & 1.2254 & 2.8542 & 1.1796 & 2.4393 \\
\hline & & & $(0.947)$ & $(0.925)$ & $(0.943)$ & $(0.938)$ & $(0.941)$ & $(0.929)$ \\
\hline \multirow[t]{6}{*}{40} & 30 & $\left(10,29^{0}\right)$ & 1.0117 & 2.2732 & 1.0123 & 2.0906 & 1.0107 & 2.0758 \\
\hline & & & $(0.952)$ & $(0.948)$ & $(0.939)$ & $(0.941)$ & $(0.947)$ & $(0.934)$ \\
\hline & & $\left(10^{0}, 10^{1}, 10^{0}\right)$ & 1.0184 & 2.3672 & 1.0189 & 2.3766 & 1.0172 & 2.0889 \\
\hline & & & $(0.957)$ & $(0.940)$ & $(0.963)$ & $(0.949)$ & $(0.952)$ & $(0.945)$ \\
\hline & & $\left(29^{0}, 10\right)$ & 1.2089 & 2.7861 & 1.2091 & 2.7936 & 1.1453 & 2.3296 \\
\hline & & & $(0.956)$ & $(0.936)$ & $(0.948)$ & $(0.939)$ & $(0.954)$ & $(0.948)$ \\
\hline \multirow[t]{6}{*}{50} & 40 & $\left(10,39^{0}\right)$ & 0.8307 & 1.5864 & 0.8592 & 1.6017 & 0.7871 & 1.4843 \\
\hline & & & $(0.945)$ & $(0.937)$ & $(0.946)$ & $(0.925)$ & $(0.941)$ & $(0.929)$ \\
\hline & & $\left(15^{0}, 10^{1}, 15^{0}\right)$ & 0.8625 & 1.6346 & 0.9125 & 1.6364 & 0.8576 & 1.5379 \\
\hline & & & $(0.952)$ & $(0.939)$ & $(0.961)$ & $(0.949)$ & $(0.951)$ & $(0.945)$ \\
\hline & & $\left(39^{0}, 10\right)$ & 0.9251 & 1.7454 & 0.9635 & 1.7849 & 0.9131 & 1.6562 \\
\hline & & & $(0.961)$ & $(0.948)$ & $(0.952)$ & $(0.943)$ & $(0.954)$ & $(0.945)$ \\
\hline
\end{tabular}

Note: Corresponding to each scheme, the first figure represents the average confidence interval and credible interval lengths, with the corresponding coverage percentage reported below it in parentheses.

\section{Conclusion}

In this article, we have considered the maximum likelihood (ML), and Bayes estimates for the parameters of the compound Rayleigh distribution $\operatorname{CRD}(\alpha, \beta)$ using progressive type-II censoring scheme. Also, we develop different confidence intervals, namely the confidence intervals obtained by using asymptotic distributions of the MLEs, two different bootstrap confidence intervals and the symmetric credible intervals for the parameters of the $\operatorname{CRD}(\alpha, \beta)$. A simulation study was conducted to examine and compare the performance of the proposed methods for different sample sizes, and different censoring schemes. From the results, we observe the following.

(i) From the results obtained in Tables 2-5. It can be seen that the performance of the Bayes estimators with respect to the noninformative prior (prior 0) is quite close to that of the MLEs. 
(ii) Tables 2-5 report the results based on informative prior, (prior 1) also in these case the results based on using the Gibbs sampling procedure are quite similar in nature when comparing the Bayes estimators based on informative prior clearly shows that the Bayes estimators based on prior 1 perform better than the MLEs, in terms of both MSEs and lengths of the confidence interval and credible interval.

(iii) From Tables 2-5, comparing the schemes $(n-m, \ldots, 0)$ and $(0, \ldots, n-m)$, it is clear that the MSEs, and average confidence interval lengths, credible interval lengths of the MLEs and Bayes estimators for both parameters are greater for the censoring scheme $(0, \ldots, n-m)$ than the censoring scheme $(n-m, \ldots, 0)$. This may not be very surprising, because the expected duration of the experiments is greater for censoring scheme $(n-m, \ldots, 0)$ than for the censoring scheme $(0, \ldots, n-m)$. Thus the data obtained by the censoring scheme $(n-m, \ldots, 0)$ would be expected to provide more information about the unknown parameters than the data obtained by censoring scheme $(0, \ldots, n-m)$.

\section{References}

[1] E. K. AL-Hussaini and Z. F. Jaheen, Approximate Bayes estimators applied to the Burr model, Communications in Statistics Theory and Methods 23 (1994) 99-121.

[2] E. K. AL-Hussaini and Z. F. Jaheen, Bayesian prediction bounds for the Burr XII Model, Communications in Statistics Theory and Methods 24 (1995) 1829-1842.

[3] N. Balakrishnan, Progressive censoring methodology: an appraisal (with discussions), TEST 16(2) (2007) 211-259.

[4] N. Balakrishnan and R. Aggrawala, Progressive censoring, theory, methods and applications (Birkhauser, Boston, 2000).

[5] N.Balakrishnan, N. Kannan, C.T. Lin and S.J.S. Wu, Inference for the extreme value distribution under progressive type-II censoring, Journal of Statistical Computation and Simulation 25 (2004) 25-45.

[6] N. Balakrishnan and R. A. Sandhu, A simple simulation algorithm for generating progressively type-II censored samples, American Statistics 49 (1995) 229-230.

[7] U Balasooriya and N. Balakrishnan, Reliability sampling plan for log-normal distribution, IEEE Transactions on Reliability 49 (2000) 199-203.

[8] P. Basak, I. Basak and N. Balakrishnan, Estimation for the threeparameter lognormal distribution based on progressively censored data, Computational Statistics and Data Analysis 53 (2009) 3580-3592.

[9] A. Bekker, J. Roux and P. Mostert, A generalization of the compound Rayleigh distribution: using a Bayesian methods on cancer survival times, Communications in Statistics Theory and Methods 29(7) (2000) 1419-1433.

[10] B. Efron, The Bootstrap and other resampling plans, In: CBMS-NSF Regional Conference Seriesin, Applied Mathematics, SIAM, (Philadelphia, PA,1982).

[11] W. R. Gilks, S. Richardson and D. J. Spiegelhalter, Markov chain Monte Carlo in Practices, (Chapman and Hall, London, 1996).

[12] EJ Green, FA Roesch, AFM Smith and WE. Strawderman, Bayesian estimation for the three-parameter Weibull distribution with tree diameter data Biometrica 50 (1994) 254-269.

[13] A. Gupta , B. Mukherjee and SK. Upadhyay, A Bayes study using Markov Chain Monte Carlo simulation, Reliability Engineering \& System Safety 93 (2008) 1434-1443.

[14] P. Hall, Theoretical comparison of Bootstrap confidence intervals, Annals of Statistics 16 (1988) 927953.

[15] W. K. Hastings, Monte Carlo sampling methods using Markov chains and their applications, Biometrika 57 (1970) 97-109.

[16] C. Kim, J. Jung and Y. Chung, Bayesian estimation for the exponentiated Weibull model under type II progressive censoring, Statistical Papers 52(1) (2011) 53-70. 
[17] MT. Madi and MZ. Raqab, Bayesian inference for the generalized exponential distribution based on progressively censored data, Communications in Statistics - Theory and Methods 38 (2009) 2016-29.

[18] M. A. W. Mahmoud, A. A. Soliman, A. H. Abd Ellah and R. M. EL-Sagheer, Markov chain Monte Carlo to study the estimation of the coefficient of variation, International Journal of Computer Applications 77 (2013) 31-37.

[19] N. Metropolis, AW. Rosenbluth, MN. Rosenbluth, AH. Teller and E. Teller, Equations of state calculations by fast computing machine, Journal of Chemical Physics 21 (1953) 1087-1091.

[20] H.K.T. Ng, Parameter estimation for a modeled Weibull distribution for progressively type-II censored samples, IEEE Transactions on Reliability 54(3) (2005) 374-380.

[21] A. F. M Smith and G. O. Roberts, Bayesian computation via Gibbs sampling and related Markov Chain Monte Carlo methods (with discussion), Journal of the Royal Statistical Society, Series B 55 (1993) $3-24$.

[22] A. A. Soliman, Estimation of parameters of life from progressively censored data using Burr-XII Model, IEEE Transactions on Reliability 54 (2005) 34-42. 